\title{
A CHARACTER THEORETIC APPROACH TO EMBEDDINGS OF ROOTED MAPS IN AN ORIENTABLE SURFACE OF GIVEN GENUS
}

\author{
D. M. JACKSON AND T. I. VISENTIN
}

\begin{abstract}
The group algebra of the symmetric group and properties of the irreducible characters are used to derive combinatorial properties of embeddings of rooted maps in orientable surfaces of arbitrary genus. In particular, we show that there exists, for each genus, a correspondence between the set of rooted quadrangulations and a set of rooted maps of all lower genera with a distinguished subset of vertices.
\end{abstract}

\section{INTRODUCTION}

Let $\mathscr{G}$ be a connected (unlabeled) graph with a finite number, $n$, of edges in which each edge is given a direction and is labeled uniquely with an integer between 1 and $n$. Loops and multiple edges are allowed. The symbols $e^{+}$ and $e^{-}$, respectively, denote the origin and terminus of the edge $e$. Let $\Sigma$ be a closed oriented surface without boundary. An embedding of $\mathscr{G}$ in $\Sigma$ is a continuous injective function $\varepsilon: \mathscr{G} \rightarrow \Sigma$. Two embeddings $\varepsilon, \varepsilon^{\prime}$ are equivalent if there is an orientation-preserving homeomorphism $\psi: \Sigma \rightarrow \Sigma$ such that $\psi \varepsilon=\varepsilon^{\prime}, \psi \varepsilon(e)=\varepsilon^{\prime}(e)$, and $\psi \varepsilon\left(e^{+}\right)=\varepsilon^{\prime}\left(e^{+}\right)$, for all $e \in\{1, \ldots, n\}$; that is, $\psi$ respects labeling and directing of edges. A map with associated graph $\mathscr{G}$ is an embedding of $\mathscr{G}$ in $\Sigma$. The deletion of $\mathscr{G}$ separates $\Sigma$ into regions homeomorphic to open discs, called the faces of the map, and the number of edges bordering a face is called its degree. A p-face-regular map is a map in which each face has degree $p$. In particular, 3- and 4-face-regular maps are called triangulations and quadrangulations, respectively. A map has genus $g$ if it is embeddable in an orientable surface of genus $g$ and no lower. By the Euler-Poincaré formula, the genus, $g$, of $\mathscr{G}$ is given by $2-2 g=i-k+j$ where $\mathscr{G}$ has $i$ vertices, $k$ edges, and $j$ faces. Fuller details are found in [9]. Figure 1 gives a triangulation with two vertices and four faces in the torus, its number of rootings (in parentheses), and an embedding of it in a polygonal

Received by the editors December 1, 1988.

1980 Mathematics Subject Classification (1985 Revision). Primary 05A15, 20C15; Secondary 57 N37.

This work was supported by a grant from the Natural Sciences and Engineering Research Council of Canada (A-8235). 


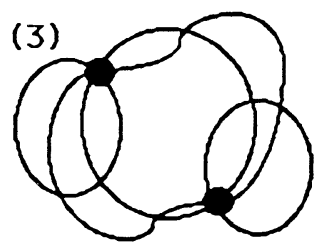

(i)

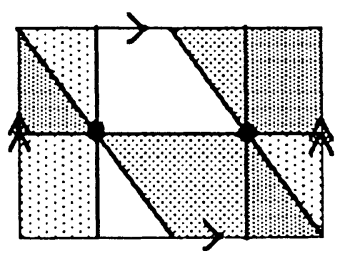

(ii)

Figure 1. A map and its embedding in the torus

representation of the torus in which faces are the union of similarly shaded areas.

This work has two purposes. The first is to investigate the connections between the group algebra of the symmetric group, character theory, and embeddings of maps in orientable surfaces. The second is to use this information to derive some new results about embeddings. The context is an enumerative one, so these results concern bijections between classes of rooted maps. These hold for all genera, thereby giving extensions to the classical results for the sphere. It is shown that there is a bijection between rooted maps and rooted quadrangulations (Corollary 5.2). A combinatorial description of this bijection would clearly be of considerable interest. Two further bijections are given in [14], a continuation of the methods of this paper.

Maps, their properties, and, in particular, their enumeration have been studied extensively. It is clear that their enumeration is made difficult by the presence of automorphisms in the associated graph and by the restrictions imposed by the presence of the surface. The automorphisms can be destroyed (restricted to the identity) by distinguishing a mutually incident vertex, the root vertex, and edge, the root edge, in the map, that is, by rooting the map. This is represented by a directed edge with the convention that the root vertex is its origin. Although rooting is an artifice, asymptotically for many natural classes of maps, the maps have no symmetries, so in these classes the ratio of the numbers of rooted to unrooted maps on $n$ edges approaches [4] the number of distinct rootings, for large $n$. This ratio is $2 n$, the number $(n)$ of ways of selecting an edge and its direction ( 2 ways), and is $4 n$ if the orientation of the surface is taken into account. The enumeration of maps is therefore often recoverable from that of rooted maps, at least asymptotically for $n$.

Little attention has been given to the use of the group algebra of the symmetric group and the associated character theory to elicit further information about embeddings. The group algebra is relevant since an embedding of a rooted map in an orientable surface is uniquely defined by a permutation, called a rotation system, encoding the vertices in its cycles. The cycles in the product of this with a particular fixed-point free involution determine the faces in the minimal embedding, and thence the genus. Drouffe [5, Appendix] briefly referred to the use of characters and made some calculations for maps with one face. 
To make this paper self-contained we have stated essential results from combinatorial topology [9] and character theory [17] without proof, and the reader is referred to the indicated sources for further details. In general, we have remained consistent with Macdonald's [16] notational conventions for symmetric functions. For graph theoretic terminology, of which we have used a minimal amount, we have followed [9].

The combinatorial connections between the group algebra and embeddings of rooted maps and between rotation systems and transitive rotation systems are given in $\S 2$. Expressions for the generating functions for classes of rooted maps indexed by the number of vertices, edges, and faces and for the associated rotation systems are given in terms of central elements corresponding to conjugacy classes in $\S 3$. Each of these generating functions determines the other, but it is more convenient to work with rotation systems. The generating function for rotation systems is expressed in terms of the irreducible characters of the symmetric group evaluated at elements all of whose cycles have the same length. A factorization of these values into irreducible characters evaluated at elements of the same type in symmetric groups of lower orders is given in $\S 4$. A factorization of a class of rotation systems is given in $\S 5$, and this leads to a simple linear relationship between the generating function for rooted quadrangulations and the generating function for all rooted maps.

The following notation is used extensively in the statements of the main results. The notation required for more local purposes appears in the introductory paragraphs of the appropriate section. A partition $\theta=\left(\theta_{1}, \theta_{2}, \ldots\right)$ is a (possibly infinite) sequence of integers such that $\theta_{1} \geq \theta_{2} \geq \cdots \geq 0$, and their sum is called the weight, $|\theta|$, of $\theta$. We write $\theta \vdash n$ to indicate that $\theta$ is a partition of $n$. The nonzero elements of $\theta$ are called its parts, and their number, $l(\theta)$, is the length of $\theta$. If $i$ occurs $m_{i}$ times as a part in $\theta$, we write $\theta=\left[1^{m_{1}} 2^{m_{2}} \ldots\right]$, suppressing $i^{m_{i}}$ if $m_{i}=0$. The conjugate, $\tilde{\theta}$, of $\theta$ is $\left(\theta_{1}^{\prime}, \theta_{2}^{\prime}, \ldots\right)$, where $\theta_{i}^{\prime}$ is the number of parts of $\theta$ which are greater than or equal to $i, i=1, \ldots, \theta_{1}$. Clearly $\tilde{\theta} \vdash|\theta|$. For $\mathscr{A} \subseteq \mathscr{N}=\{1,2, \ldots\}, \Pi_{\mathscr{A}}$ denotes the set of all partitions with no part not in $\mathscr{A}$. A sequence $\left(\theta^{(1)}, \ldots, \theta^{(k)}\right)$ of partitions whose weights sum to $n$ is called a multipartition of $n$, and the set of all multipartitions of weight $n$ with $k$ components is denoted by $\Pi_{k, n}$.

The set $\{\theta \vdash n\}$ is a natural index set for the conjugacy classes of $\mathfrak{S}_{n}$, the symmetric group of $n$ symbols. The cycle-type of $\pi \in \mathscr{C}_{\theta}$, a conjugacy class of $\mathfrak{S}_{n}$, is $\left[1^{m_{1}} 2^{m_{2}} \cdots\right] \vdash n$, where $\pi$ has $m_{i}$ cycles of length $i$ for $i=1,2, \ldots$. The natural indexing is such that $\theta=\left[1^{m_{1}} 2^{m_{2}} \ldots\right]$. Thus $l(\theta)$ is the number of cycles of $\pi$. The order of $\mathscr{C}_{\theta}$ is $h^{\theta}=n ! g(\theta)$, where $g(\theta)=$ $\prod_{i \geq 1}\left(i^{m_{i}} m_{i} !\right)^{-1}$. The group algebra of $\mathfrak{S}_{n}$ over the complex field $\mathbf{C}$ is denoted by $\mathbf{C S}_{n}$. Multiplication of permutations is carried out from right to left. A permutation in $\mathfrak{S}_{k p}$ is $p$-semiregular if its cycle-type is $\left[p^{k}\right]$. Occasionally, we drop the prefix and refer to such permutations as semiregular. 
$\chi^{\theta}$ denotes the irreducible (ordinary) character associated with $\mathscr{C}_{\theta}$ and $\chi_{\alpha}^{\theta}$ denotes its value at any element of $\mathscr{C}_{\alpha}$ where $\alpha, \theta \vdash N$. For convenience, we adopt the convention that the character associated with the empty partition is identically 1 and that $\chi_{\alpha}^{\theta}=0$ if $|\theta| \neq|\alpha|$. The degree, $f^{\theta}$, of the irreducible representation indexed by $\theta$ is equal to $\chi_{\left[1^{N}\right]}^{\theta}$.

If $\mathbf{A}$ is an $r \times s$ matrix whose $(i, j)$-element is $a_{i j}$, we write $\mathbf{A}=\left[a_{i j}\right]_{r \times s}$. When $r=s$, the determinant $|\mathbf{A}|$ of $\mathbf{A}$ is denoted by $\left\|a_{i j}\right\|$ (or by $\left\|\left[a_{i j}\right]\right\|_{r \times r}$ ).

If $\left\{a_{1}, \ldots, a_{r}\right\}$ is a set of real numbers, then we write $\left\{b_{1}, \ldots, b_{r}\right\}=$ $\left\{a_{1}, \ldots, a_{r}\right\}_{\leq}$to signify that $\left\{b_{1}, \ldots, b_{r}\right\}=\left\{a_{1}, \ldots, a_{r}\right\}$ and $b_{1} \leq \cdots \leq b_{r}$.

The rising and falling factorial functions are, respectively, $(x)^{(n)}=$ $x(x+1) \cdots(x+n-1)$ and $(x)_{n}=x(x-1) \cdots(x-n+1)$. Finally, $H_{\theta}(x)$ denotes the polynomial $\prod_{1 \leq i \leq l(\theta)}(x-i+1)^{\left(\theta_{i}\right)}$.

\section{GRAPH EMBEDdiNGS AND THE GROUP ALGEBRA}

The set of origins $e^{+}$and termini $e^{-}$of edges in the edge-set $E(\mathscr{G})=$ $\{1, \ldots, n\}$ of $\mathscr{G}$ is called the directed edge set of $\mathscr{G}$. Each vertex $v$ of $\mathscr{G}$ is represented as a subset of the directed edge set, so $e^{+}$is in the subset if and only if $v$ is the origin of $e$, and $e^{-}$is in the subset if and only if $v$ is the terminus of $e$. These subsets partition the directed edge set. For each vertex $v$ of $\mathscr{G}$, we specify a cyclic list of the directed edges encountered in traversing the boundary of a small disc, centered at $v$, in the sense specified by the orientation of $\Sigma$. We shall fix this sense to be anticlockwise. This partition of the directed edge set into cyclic lists is called a rotation system, and it represents a permutation on the set $\left\{1^{+}, 1^{-}, \ldots, n^{+}, n^{-}\right\}$. By a generalization of a theorem of Schoenflies [11] it is known that, for every embedding of $\mathscr{G}$ in $\Sigma, \mathscr{G}$ is contained in the 1 -skeleton of a triangulation of this surface. This permits the analysis of graph embeddings by combinatorial methods.

Theorem 2.1. Every rotation system for a connected graph $\mathscr{G}$ induces, up to equivalence of embeddings, a unique embedding of $\mathscr{G}$ in $\Sigma$. Conversely, every embedding of $\mathscr{G}$ in $\Sigma$ induces a unique transitive rotation system.

This one-to-one correspondence, sometimes called the Edmonds embedding theorem [6], was given originally in dual form by Heffter [10] and has been generalized to graphs with loops and multiple edges by Gross and Alpert [8]. An important consequence of this theorem is that the faces of the embeddings can be determined by algebraic means.

Proposition 2.2. Let $\nu$ be the rotation system of a graph $\mathscr{G}$ on $n$ edges. Let $\epsilon_{n}=\left(1^{+} 1^{-}\right)\left(2^{+} 2^{-}\right) \cdots\left(n^{+} n^{-}\right)$be the fixed permutation describing, in its cycles, the edges of $\mathscr{G}$. Then the cycles of $\nu \epsilon_{n}$ list the directed edges encountered in traversing the boundary of the faces of the embedding in a direction consistent with the orientation of $\Sigma$. 


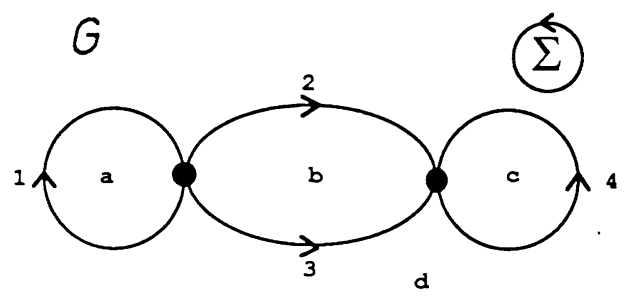

FIGURE 2. A transitive rotation system

For example, the rotation system corresponding to the graph in Figure 2 is $\nu=\left(1^{+} 3^{+} 2^{+} 1^{-}\right)\left(2^{-} 3^{-} 4^{+} 4^{-}\right)$. From Proposition 2.2, its faces are therefore given by $\phi=\nu \epsilon_{4}=\left(1^{+}\right)\left(2^{+} 3^{-}\right)\left(4^{-}\right)\left(1^{-} 3^{+} 4^{+} 2^{-}\right)$. The cycle $\left(2^{+} 3^{-}\right)$represents the face labeled $b$ in Figure 2 and indicates that edge 2 is encountered in its positive direction followed by the edge 3 in its negative direction in traversing the boundary of this face in the direction consistent with the orientation of $\Sigma$. In this embedding there are four faces, so the genus of this map is zero.

The number of rooted maps is readily deducible from the number of rotation systems.

Lemma 2.3. For each rooted map on $n$ edges there are $t(n)=(n-1) ! 2^{n-1}$ distinct (transitive) rotation systems.

Proof. The nonroot edges can be labeled in $(n-1)$ ! ways, and each such edge can be directed in two ways.

Not every permutation $\nu \in \mathfrak{S}_{2 n}$ corresponds to a rotation system for an embedding of a graph with $n$ edges. If $\nu$ and $\epsilon_{n}$ do not generate $\mathfrak{S}_{2 n}$, then the associated graph $\mathscr{G}$ is not connected. However, the following corollary of Theorem 2.1 gives a combinatorial description of every element of $\mathfrak{S}_{2 n}$ in terms of transitive substructures.

To this end, for $\rho, \sigma \in \mathfrak{S}_{N}$, let $\|\rho, \sigma\|$ denote the number of orbits of the group generated by $\rho$ and $\sigma$. For any $\mathscr{I}=\left\{i_{1}, \ldots, i_{k}\right\} \subseteq \mathscr{N}_{n}$, let $\mathscr{P}_{\mathscr{I}}$ be the set of all permutations of the symbols $i_{1}^{+}, i_{1}^{-}, \ldots, i_{k}^{+}, i_{k}^{-}$and let $\epsilon_{\mathscr{J}}=$ $\left(i_{1}^{+} i_{1}^{-}\right) \cdots\left(i_{k}^{+} i_{k}^{-}\right) \in \mathscr{P}_{\mathscr{J}}$. Let $\mathscr{A} \subseteq\{1,2, \ldots\}$ and let $\mathscr{P}_{\mathscr{I}}^{\mathscr{A}}$ be the subset of $\mathscr{P}_{\mathscr{I}}$ consisting of all permutations having no cycles of length not in $\mathscr{A}$. Let $\mathscr{T}_{\mathscr{J}}^{\mathscr{A}}=\left\{\sigma \in \mathscr{P}_{\mathscr{I}}^{\mathscr{A}}:\left\|\epsilon_{\mathscr{I}}, \sigma\right\|=1\right\}$.

Corollary 2.4. $\mathscr{P}_{\mathscr{N}_{n}}^{\mathscr{A}}$ is in 1-1 correspondence with

$$
\mathscr{Q}_{n}^{\mathscr{A}}=\bigcup_{m=1}^{n}\left\{\left\{\sigma_{1}, \ldots, \sigma_{m}\right\}: \sigma_{j} \in \mathscr{T}_{\mathcal{F}_{j}}^{\mathscr{A}}, j=1, \ldots, m ; \mathscr{I}_{1} \dot{\cup} \ldots \dot{\cup} \mathscr{I}_{m}=\mathscr{N}_{n}\right\}
$$

Proof. For $\pi \in \mathscr{P}_{\mathscr{N}_{n}}^{\mathscr{A}}$, let $\mathscr{G}_{\pi}$ be the group generated by $\pi$ and $\epsilon_{n}$. Suppose that $\mathscr{G}_{\pi}$ has orbits $\mathscr{I}_{1}, \ldots, \mathscr{I}_{m}$. Let $\pi_{j}$ be the permutation formed by the product 
of the cycles of $\pi$ which act on $\mathscr{I}_{j}$, for $j=1, \ldots, m$. Then $\left\{\pi_{1}, \ldots, \pi_{m}\right\} \in$ $\mathscr{Q}_{n}^{\mathscr{A}}$. Let $\psi$ be the mapping

$$
\psi: \mathscr{P}_{\mathscr{N}_{n}}^{\mathscr{A}} \rightarrow \mathscr{Q}_{n}^{\mathscr{A}}: \pi \mapsto\left\{\pi_{1}, \ldots, \pi_{m}\right\} .
$$

Let $\left\{\sigma_{1}, \ldots, \sigma_{m}\right\} \in \mathscr{Q}_{n}^{\mathscr{A}}$. Since $\sigma_{1}, \ldots, \sigma_{m}$ all act on different sets, the product $\sigma_{1} \cdots \sigma_{m}$ is uniquely determined, and $\sigma \in \mathscr{P}_{\mathscr{N}_{n}}^{\mathscr{A}}$. It is a straightforward matter to check that $\psi$ is bijective.

In view of Corollary 2.4, each permutation of $\mathfrak{S}_{2 n}$ is a rotation system for an unordered collection of graph embeddings. Proposition 2.2 can be applied in this more general setting to show that the cycles of $\nu \epsilon_{n}$ describe the faces of the embeddings of the unordered collection.

We conclude this section with some combinatorial facts about $\mathbf{C S}_{N}$ which, when combined with observations on the connection between rooted maps and rotation systems, will enable us to enumerate classes of rooted maps, at least in principle.

Each element of $\mathbf{C S}_{N}$ can be expressed uniquely as a finite linear combination of elements in $\mathfrak{S}_{N}$ with coefficients in $\mathbf{C}$. The product of two elements in $\mathrm{CS}_{N}$ is taken distributively with respect to the product on $\mathfrak{S}_{N}$. Let $\mathrm{K}_{\theta}$ be the formal sum of all elements of $\mathscr{C}_{\theta}$ for $\theta \vdash N$. Then $\left\{\mathrm{K}_{\theta}: \theta \vdash N\right\}$ is a basis for the center of $\mathrm{CS}_{N}$, so the coefficient of $\mathrm{K}_{\theta}$ in the product $\mathrm{K}_{\alpha} \mathrm{K}_{\beta}$, for $\alpha, \beta \vdash n$, is uniquely defined. This is denoted by $\left[\mathrm{K}_{\theta}\right] \mathrm{K}_{\alpha} \mathrm{K}_{\beta}$, and, in the present context, it has the following useful combinatorial interpretation [12, Proposition 2.2].

Proposition 2.5. Let $\alpha, \beta, \theta \vdash N$. Then the number of ways of expressing an arbitrary element $c \in \mathscr{C}_{\theta}$ as $c=$ ab with $(a, b) \in \mathscr{C}_{\alpha} \times \mathscr{C}_{\beta}$ is $\left[\mathrm{K}_{\theta}\right] \mathrm{K}_{\alpha} \mathrm{K}_{\beta}$. Moreover, this is equal to $\left[\mathrm{K}_{\beta}\right] \mathrm{K}_{\alpha} \mathrm{K}_{\theta}$.

We therefore have the following basic enumerative result for rotation systems.

Lemma 2.6. Let $n$ be a nonnegative integer and let $\nu, \phi \vdash 2 n$. Then

$$
\left|\mathscr{C}_{\nu} \epsilon_{n} \cap \mathscr{C}_{\phi}\right|=\frac{h^{\phi}}{h^{\left[2^{n}\right]}}\left[\mathrm{K}_{\theta}\right] \mathrm{K}_{\nu} \mathrm{K}_{\left[2^{n}\right]} .
$$

Proof.

$$
\begin{aligned}
\left|\mathscr{C}_{\nu} \epsilon_{n} \cap \mathscr{C}_{\phi}\right| & =\sum_{\substack{(a, b) \in \mathscr{C}_{\nu} \times\left\{\epsilon_{n}\right\} \\
a b \in \mathscr{C}_{\phi}}} 1=\frac{1}{h^{\left[2^{n}\right]}} \sum_{\substack{(a, b) \in \mathscr{C}_{\nu} \times \mathscr{C}_{\left[2^{n}\right]} \\
a b \in \mathscr{C}_{\phi}}} 1 \\
& =\frac{h^{\phi}}{h^{\left[2^{n}\right]}} \sum_{\substack{(a, b) \in \mathscr{C}_{\nu} \times \mathscr{C}_{\left[2^{n}\right]} \\
a b=c}} 1
\end{aligned}
$$

for an arbitrary but fixed $c \in \mathscr{C}_{\phi}$. The result follows from Proposition 2.5.

\section{GenERATING FUNCTIONS FOR ROOTED MAPS}

The following conventions for generating functions are adopted in the interests of brevity. Let $\mathbf{m}=\left(m_{1}, \ldots, m_{p}\right), \mathbf{n}=\left(n_{1}, \ldots, n_{q}\right)$ be sequences 
of nonnegative integers, empty if $p=0$ or $q=0$. Let $\mathbf{x}=\left(x_{1}, \ldots, x_{p}\right)$, $\mathbf{y}=\left(y_{1}, \ldots, y_{q}\right)$ be sequences of commuting indeterminates. Then $\mathbf{m} ! \mathrm{de}-$ notes $m_{1} ! \cdots m_{p} !$, and $\mathbf{x}^{\mathbf{m}}$ denotes $x_{1}^{m_{1}} \cdots x_{p}^{m_{p}}$. Let $\mathbf{0}_{p}=(0, \ldots, 0)$, with $p$ components, and let $\mathbf{x} \geq \mathbf{m}$ mean that $x_{i} \geq m_{i}$ for $i=1, \ldots, p$. For brevity, we write $\mathbf{m} \geq \mathbf{0}$ instead of $\mathbf{m} \geq \mathbf{0}_{p}$, the suppressed suffix being understood from the context.

A generating function (series) for the sequence $(a(\mathbf{m}, \mathbf{n}) \in \mathbf{Q}: \mathbf{m}, \mathbf{n} \geq \mathbf{0})$ is $\Phi(\mathbf{x} \mid \mathbf{y})=\sum_{\mathbf{m}, \mathbf{n} \geq \mathbf{0}} a(\mathbf{m}, \mathbf{n}) \mathbf{x}^{\mathbf{m}} \mathbf{y}^{\mathbf{n}} / \mathbf{n}$ !. When $q=0$, this is denoted by $\boldsymbol{\Phi}(\mathbf{x})$. Let $\mathbf{k}=\left(k_{1}, \ldots, k_{p}\right)$ be a sequence of integers. The coefficient operator [.] is

$$
\left[\mathbf{x}^{k}\right]: \mathbf{Q}[[\mathbf{x}, \mathbf{y}]] \rightarrow \mathbf{Q}[[\mathbf{y}]]: \mathbf{\Phi} \mapsto b_{\mathbf{k}}(\mathbf{y})
$$

where $\sum_{\mathbf{m}} b_{\mathbf{m}}(\mathbf{y}) \mathbf{x}^{\mathbf{m}}$ is the image of $\mathbf{\Phi}$ under the natural isomorphism

$$
\mathbf{Q}[[\mathbf{x}, \mathbf{y}]] \rightarrow(\mathbf{Q}[[\mathbf{y}]])[[\mathbf{x}]] .
$$

For example, $[x] x y=y,\left[x y^{0}\right] x y=0$. When $a(\mathbf{m}, \mathbf{n})$ is the cardinality of a set of combinatorial objects, combinatorial considerations [7] determine the selection of $p$ and $q$ that is convenient for the particular instance. We say that $x_{i}$ marks the combinatorial feature counted by $m_{i}$.

In view of Lemma 2.3 and the Euler-Poincare formula, it is convenient to introduce the mapping $\Omega_{u}$ defined by $\Omega_{u}\left(x^{i} y^{j} z^{k} / k !\right)=x^{i} y^{j} z^{k} u^{2+k-i-j} / t(k)$, extended linearly to $\mathbf{Q}[[x, y, z]]$. We shall apply this only when $i, j, k$ count, respectively, the vertices, faces, and edges of a map, so $2+k-i-j=2 g$ where $g$ is the genus of the map. The action of $\Omega_{u}$ on $f \in \mathbf{Q}[[x, y, z]]$ can be represented differentially by

$$
\Omega_{u} f(x, y, z)=2 u^{2} z \frac{\partial}{\partial z} f\left(x u^{-1}, y u^{-1}, \frac{1}{2} z u\right) .
$$

For $\mathscr{A} \subseteq \mathscr{N}$, let $M_{\mathscr{A}}(u, x, y, z)$ be the generating function for $m_{g, i, j, k}^{\mathscr{A}}$, the number of rooted maps of genus $g$ with $i$ vertices, $j$ faces, and $k$ edges, and with no faces of degree not in $\mathscr{A}$. This is also called the genus distribution of the class of maps designated by $\mathscr{A}$. Let $R_{\mathscr{A}}(x, y \mid z)$ be the generating function for $r_{i, j, k}^{\mathscr{A}}$, the number of rotation systems $\nu \in \mathfrak{S}_{2 k}$ with $i$ cycles such that $\epsilon_{k} \nu$ has $j$ cycles, none of whose lengths are not in $\mathscr{A}$. Thus $M_{\mathscr{A}} \in$ $\mathbf{Q}[u, x, y][[z]]$, since maps with a finite number of edges are themselves finite. Similarly, $R_{\mathscr{A}} \in \mathbf{Q}[x, y][[z]]$. Note that $M_{\mathscr{N}}$ and $M_{\{4\}}$ are the generating functions for all rooted maps and rooted quadrangulations, respectively.

Proposition 3.1. $M_{\mathscr{A}}(u, x, y, z)=M_{\mathscr{A}}(u, y, x, z)$.

Proof. By duality.

It is clear from Corollary 2.4 that $\log R_{\mathscr{A}}(x, y \mid z)$ is the generating function for the corresponding set of transitive rotation systems. We may now express $M_{\mathscr{A}}$ fully in terms of the group algebra of the symmetric group. 
Theorem 3.2.

$$
\begin{gathered}
M_{\mathscr{A}}\left(u^{2}, x, y, z\right)=\Omega_{u} \log R_{\mathscr{A}}(x, y \mid z) \\
R_{\mathscr{A}}(x, y \mid z)=\sum_{n \geq 0} \frac{z^{n}}{n !} \sum_{\substack{\nu, \phi \vdash 2 n \\
\phi \in \Pi_{\mathscr{A}}}} \frac{h^{\phi}}{h^{\left[2^{n}\right]}}\left[\mathrm{K}_{\phi}\right] \mathrm{K}_{\nu} \mathrm{K}_{\left[2^{n}\right]} x^{l(\nu)} y^{l(\phi)}
\end{gathered}
$$

Proof. (1) Let $c_{i, j, n}^{\mathscr{A}}$ be the number of transitive rotation systems among those counted by $r_{i, j, n}^{\mathscr{A}}$, and let $C_{\mathscr{A}}(x, y \mid z)$ be the generating function for $\left(c_{i, j, n}^{\mathscr{A}}\right.$ : $i, j, n \geq 0)$. By Corollary 2.4, $R_{\mathscr{A}}=\exp C_{\mathscr{A}}$. But, from Lemma 2.3, $c_{i, j, n}^{\mathscr{A}}=$ $t(n) m_{g, i, j, n}^{\mathscr{A}}$ where $2 g=2+n-i-j$ so

$$
C_{\mathscr{A}}(x, y \mid z)=\sum_{i, j, n \geq 0} t(n) m_{g, i, j, n}^{\mathscr{A}} x^{i} y^{j} \frac{z^{n}}{n !}
$$

whence

$$
\Omega_{u} \log R_{\mathscr{A}}(x, y \mid z)=\Omega_{u} C_{\mathscr{A}}(x, y \mid z)=M_{\mathscr{A}}\left(u^{2}, x, y, z\right) .
$$

(2) Now $r_{i, j, k}^{\mathscr{A}}=\sum\left|\mathscr{C}_{\nu} \epsilon_{k} \cap \mathscr{C}_{\phi}\right|$ where the summation is over $\phi \vdash 2 k$ such that $l(\phi)=j, \phi \in \Pi_{\mathscr{A}}$, and $\nu \vdash 2 k$ such that $l(\nu)=i$. The result follows from Lemma 2.6.

In view of Theorem 3.2, it is sufficient to focus attention on $R_{\mathscr{A}}$ in the determination of $M_{\mathscr{A}}$. We now use properties of the center of $\mathrm{CS}_{N}$.

Since $\mathbf{C S}_{N}$ is semisimple [17], its center has a basis $\left\{\mathrm{F}_{\theta}: \theta \vdash N\right\}$ of orthogonal idempotents, which are therefore expressible in terms of $\left\{\mathrm{K}_{\theta}: \theta \vdash\right.$ $N\}$ and vice versa. These are expressed in terms of each other by $\mathrm{F}_{\alpha}=$ $N !^{-1} f^{\alpha} \sum_{\theta \vdash N} \chi_{\theta}^{\alpha} \mathrm{K}_{\theta}$ and $\mathrm{K}_{\alpha}=h^{\alpha} \sum_{\theta \vdash N} \chi_{\alpha}^{\theta} \mathrm{F}_{\theta} / f^{\theta}$ for $\alpha \vdash N$. These central idempotents can be used to express as explicit character sums the enumerative quantities of the type appearing in Lemma 2.6 and in association with various classes of maps.

Lemma 3.3.

$$
\left[\mathrm{K}_{\gamma}\right] \mathrm{K}_{\alpha} \mathrm{K}_{\beta}=\frac{1}{N !} h^{\alpha} h^{\beta} \sum_{\theta \vdash N} \frac{1}{f^{\theta}} \chi_{\alpha}^{\theta} \chi_{\beta}^{\theta} \chi_{\gamma}^{\theta} .
$$

Proof. From the above expressions connecting $\left\{\mathrm{K}_{\theta}: \theta \vdash N\right\}$, and $\left\{\mathrm{F}_{\theta}: \theta \vdash\right.$ $N\}$, and from the fact that the $\mathrm{F}_{\alpha}$ 's are orthogonal idempotents, the left-hand side is $h^{\alpha} h^{\beta} \sum_{\theta \vdash N}\left(f^{\theta}\right)^{-2} \chi_{\alpha}^{\theta} \chi_{\beta}^{\theta}\left[\mathrm{K}_{\gamma}\right] \mathrm{F}_{\theta}$, and the result follows since $\left[\mathrm{K}_{\gamma}\right] \mathrm{F}_{\theta}=$ $f^{\theta} \chi_{\gamma}^{\theta} / N !$.

Proposition 3.4.

$$
\sum_{\alpha \vdash N} h^{\alpha} \chi_{\alpha}^{\theta} x^{l(\alpha)}=f^{\theta} H_{\theta}(x) .
$$

Proof. See, for example, [13].

The next corollary gives explicit expressions for the generating functions for rotation systems, with and without conditions. 
Corollary 3.5 .

$$
\begin{aligned}
& R_{\mathscr{N}}(x, y \mid z)=\sum_{n \geq 0} \frac{z^{n}}{n !(2 n) !} \sum_{\theta \vdash 2 n} f^{\theta} \chi_{\left[2^{n}\right]}^{\theta} H_{\theta}(x) H_{\theta}(y) . \\
& R_{\mathscr{A}}(x, y \mid z)=\sum_{n \geq 0} \frac{z^{n}}{n !(2 n) !} \sum_{\phi \in \Pi_{\mathscr{A}}} h^{\phi} y^{l(\phi)} \sum_{\theta \vdash 2 n} \chi_{\phi}^{\theta} \chi_{\left[2^{n}\right]}^{\theta} H_{\theta}(x) .
\end{aligned}
$$

Proof. (1) From Theorem 3.2(2)

$$
R_{\mathscr{N}}(x, y \mid z)=\sum_{n \geq 0} \frac{z^{n}}{n !} \sum_{\nu, \phi \vdash 2 n} x^{l(\nu)} y^{l(\phi)} \frac{h^{\phi}}{h^{\left[2^{n}\right]}}\left[\mathrm{K}_{\phi}\right] \mathrm{K}_{\nu} \mathrm{K}_{\left[2^{n}\right]}
$$

and the result follows directly from Lemmas 3.3 and 3.4 after rearrangement of the sums.

(2) The proof is similar.

In principle, it can be checked that $\left[u^{2 g}\right] \Omega_{u} \log R_{\mathscr{N}}(1,1 \mid z)$ indeed gives the known results for the sphere [19] and the torus [2], with $g=0$ and $g=1$, respectively.

An explicit expression for the generating function for maps counted without regard to genus is immediately recoverable. Of course, this can be obtained directly from the embedding theorem (Theorem 2.1).

Proposition 3.6.

$$
M_{\mathscr{N}}(1, x, 1, z)=2 z \frac{\partial}{\partial z} \log \sum_{n \geq 0} \frac{1}{2^{n} n !} z^{n}(x)^{(2 n)} .
$$

Proof. From Theorem 3.2 and Corollary 3.5

$$
M_{\mathscr{N}}(1, x, 1, z)=2 z \frac{\partial}{\partial z} \log \sum_{n \geq 0} \frac{z^{n}}{2^{n} n !(2 n) !} \sum_{\theta \vdash 2 n} f^{\theta} \chi_{\left[2^{n}\right]}^{\theta} H_{\theta}(x) \prod_{1 \leq i \leq l(\theta)}(2-i)^{\left(\theta_{i}\right)} \text {. }
$$

But $\prod_{1 \leq i \leq l(\theta)}(2-i)^{\left(\theta_{i}\right)}=|\theta| ! \delta_{1, l(\theta)}$ and $\chi_{\left[2^{n}\right]}^{[2 n]}=1=f^{[2 n]}$. The result follows.

Note that $M_{\mathscr{N}}(1, x, 1, z)$ is the generating function for all rooted maps, without regard to genus, with respect to edges and vertices. When the set of such maps is counted with respect to the number of edges alone, then

$$
M_{\mathscr{N}}(1,1,1, z)=2 z \frac{\partial}{\partial z} \log \sum_{n \geq 0} \frac{(2 n) !}{2^{n} n !} z^{n}
$$

\section{Corollary 3.7.}

$$
M_{\{4\}}(1,1,1, z)=2 z \frac{\partial}{\partial z} \log \sum_{n \geq 0} \frac{(4 n) !}{16^{n} n !(2 n) !} z^{2 n} .
$$

Proof. From Theorem 3.2 and Corollary 3.5.

It is convenient to give explicitly the generating functions of rotation systems corresponding to face-regular rooted maps. 
Corollary 3.8.

(1) $k=2 m$ :

$$
R_{\{2 m\}}(x, y \mid z)=\sum_{n \geq 0} \frac{z^{m n}}{(m n) !} \frac{y^{n}}{k^{n} n !} \sum_{\theta \vdash k n} \chi_{\left[k^{n}\right]}^{\theta} \chi_{\left[2^{m n}\right]}^{\theta} H_{\theta}(x) .
$$

(2) $k$ odd:

$$
R_{\{k\}}(x, y \mid z)=\sum_{n \geq 0} \frac{z^{k n}}{(k n) !} \frac{y^{2 n}}{k^{2 n}(2 n) !} \sum_{\theta \vdash 2 k n} \chi_{\left[k^{2 n}\right]}^{\theta} \chi_{\left[2^{k n}\right]}^{\theta} H_{\theta}(x) .
$$

Proof. Direct from Corollary 3.5 .

\section{FACTORING CHARACTERS AT SEMIREGULAR ELEMENTS}

The importance of $\chi^{\theta}$ at semiregular permutations has been seen in Corollary 3.5. We now consider the question of evaluating $\chi^{\theta}$ at these permutations. For this purpose, the following facts (see, for example, [16]) are used.

Let $\left\{x_{1}, x_{2}, \ldots\right\}$ be a countably infinite set of algebraically independent commuting indeterminates and let $\Lambda$ be the ring of symmetric functions in $\mathbf{x}=\left(x_{1}, x_{2}, \ldots\right)$. Restriction to the finite case is obtained by setting $x_{k+1}=$ $x_{k+2}=\cdots=0$. The complete symmetric functions and power sum symmetric functions associated with the partition $\theta$ are $h_{\theta}=h_{\theta_{1}} h_{\theta_{2}} \cdots$ where $\sum_{r \geq 0} h_{r} t^{r}=$ $\prod_{i \geq 1}\left(1-t x_{i}\right)^{-1}$, and $p_{\theta}=p_{\theta_{1}} p_{\theta_{2}} \cdots$ where $p_{r}=x_{1}^{r}+x_{2}^{r}+\cdots$. These are related by $h_{r}=\sum_{\alpha \vdash r} g(\alpha) p_{\alpha}$. By a result of Jacobi, the $S c h u r$ symmetric function associated with $\theta$ is $s_{\theta}=\left\|h_{\theta_{i}-i+j}\right\|_{n \times n}$ for any $n \geq l(\theta)$, and, by a result of Frobenius, $s_{\theta}=\sum_{\alpha \vdash|\theta|} g(\alpha) \chi_{\alpha}^{\theta} p_{\alpha}$. The sets $\left\{h_{\theta}\right\},\left\{p_{\theta}\right\},\left\{s_{\theta}\right\}$ each afford a basis of $\Lambda$, so, in particular, the coefficient operator $\left[p_{\theta}\right]$ on $\Lambda$ is well-defined and acts linearly. The $p_{i}$ are algebraically independent, as are the $h_{i}$.

Furthermore, we need to construct an explicit bijection between two particular sets of partitions whose roles will become clear when they are used, for example, in Lemma 4.8. The bijection is denoted by $\Delta_{k}$ and is given in Lemma 4.6. It is preceded by preparatory results of an apparently unavoidably technical nature.

Definition 4.1. Let $k, n$ be positive integers. For $\alpha=\left(\alpha_{1}, \alpha_{2}, \ldots\right) \vdash k n$ we define $\alpha^{\wedge}=\left(\alpha^{(1)}, \ldots, \alpha^{(k)}\right)$ by

(1) $\left\{\eta_{j, 1}, \ldots, \eta_{j, m_{j}}\right\}=\left\{i: \alpha_{i}-i \equiv-j \bmod k\right\}_{<}$for $j=1, \ldots, k$,

(2) $\alpha^{(j)}=\left(\alpha_{1}^{(j)}, \ldots, \alpha_{m_{j}}^{(j)}\right)$ for $j=1, \ldots, k$,

(3) $\alpha_{i}^{(j)}=\frac{1}{k}\left(\alpha_{\eta_{j, i}}-\eta_{j, i}+j\right)+i-1$ for $i=1, \ldots, m_{j}$.

If $m_{j}=\lceil(l(\alpha)-j+1) / k\rceil$ for all $j=1, \ldots, k$, then $\alpha$ is said to be $k$ balanced. Let $\mathscr{B}_{k, n}$ be the set of all $k$-balanced partitions of $k n$. Clearly, for $\alpha$ with $l(\alpha)=q k+r$ where $q, r$ are integers and $0 \leq r<k, \alpha$ is $k$-balanced if $m_{1}=\cdots=m_{r}=q+1$ and $m_{r+1}=\cdots=m_{k}=q$. 
Proposition 4.2. If $\alpha \in \mathscr{B}_{k, n}$ then $\alpha^{\wedge} \in \Pi_{k, n}$.

Proof. From Definition 4.1(1), $\alpha_{i}^{(j)}$ is an integer and $\alpha_{1}^{(j)} \geq \cdots \geq \alpha_{m_{j}}^{(j)}$. But $\alpha \in \mathscr{B}_{k, n}$, so

$$
\begin{aligned}
\alpha_{m_{j}}^{(j)} & =\frac{1}{k}\left(\alpha_{\eta_{j, m_{j}}}-\eta_{j, m_{j}}+j\right)+m_{j}-1 \\
& \geq \frac{1}{k}(1-l(\alpha)+j)+\left[\frac{1}{k}(l(\alpha)-j+1)\right\rceil-1 \\
& \geq\left\lceil\frac{1}{k}(l(\alpha)-j+1)\right]-\frac{1}{k}(l(\alpha)-j+1)+\frac{2}{k}-1 \geq 0
\end{aligned}
$$

since $\alpha_{m_{j}}^{(j)}$ is an integer. Thus $\alpha^{\wedge}$ is a multipartition.

Moreover, $\left|\alpha^{\wedge}\right|=k^{-1} \sum_{j=1}^{k} \sum_{i=1}^{m_{j}}\left\{\alpha_{\eta_{j, i}}-\eta_{j, i}+k(i-1)+j\right\}$. But $k(i-1)+j$ and $\eta_{j, i}$ take each value in $\{1,2, \ldots, l(\alpha)\}$ exactly once for each $(i, j)$ in the indicated range. Thus the second and third sums together contribute zero to the summation, whence $\left|\alpha^{\wedge}\right|=|\alpha| / k=n$ and so $\alpha^{\wedge} \in \Pi_{k, n}$.

The following proposition follows immediately from the proof of Proposition 4.2 and, although it seems obvious, it plays a subtle and important role in Lemma 4.8 .

Proposition 4.3. If $\theta$ is $k$-balanced, then $k$ divides $|\theta|$.

Definition 4.4. Let $k, n$ be positive integers. For $\beta=\left(\beta^{(1)}, \beta^{(2)}, \ldots, \beta^{(k)}\right) \in$ $\Pi_{k, n}$ we define $\beta^{\vee}=\left(a_{1}+1, \ldots, a_{m}+m\right)$ by

(1) $q+1=\max \left\{l\left(\beta^{(j)}\right): 1 \leq j \leq k\right\}, r=\max \left\{j: l\left(\beta^{(j)}\right)=q+1\right\}$;

(2) $m_{1}=\cdots=m_{r}=q+1, m_{r+1}=\cdots=m_{k}=q$;

(3) $\left\{a_{1}, \ldots, a_{m}\right\}=\left(A^{(1)} \cup \cdots \cup A^{(k)}\right)_{>}$ where $A^{(j)}=\left\{a_{j, 1}, \ldots, a_{j, m_{j}}\right\}$ and $a_{j, i}=k\left(\beta_{i}^{(j)}-i+1\right)-j$ for $j=1, \ldots, k$ with the convention that $\beta_{i}^{(j)}=0$ for $i>l\left(\beta^{(j)}\right)$.

Proposition 4.5. If $\beta \in \Pi_{k, n}$ then $\beta^{\vee} \in \mathscr{B}_{k, n}$.

Proof. $A^{(j)}$ contains only integers congruent to $-j \bmod k$ so the $A^{(j)}$ are pairwise disjoint, whence $m=k q+r$. Moreover, $a_{j, 1}>\cdots>a_{j, m_{j}}$ for $j=1, \ldots, k$.

Now $a_{1}>\cdots>a_{m}$ so $a_{1}+1 \geq \cdots \geq a_{m}+m$. Consider $a_{m}+m=$ $k\left(\beta_{m_{j}}^{(j)}-m_{j}+1\right)-j+m$ for some $1 \leq j \leq k$. There are three cases to consider.

Case $1(j<r)$. Then $a_{m}+m \geq k(0-(q+1)+1)-j+q k+r=r-j>0$.

Case $2(j=r)$. Then $\beta_{m_{j}}^{(j)} \geq 1$ so $a_{m}+m \geq k(1-(q+1)+1)-r+q k+r=k>0$.

Case $3(j>r)$. Then $a_{m}+m \geq k(0-q+1)-j+q k+r=k-j+r>0$. 
We conclude that $\beta^{\vee}$ is a partition and that $l\left(\beta^{\vee}\right)=q k+r=m$. Finally,

$$
\begin{aligned}
\left|\beta^{\vee}\right| & =\sum_{i=1}^{m}\left(a_{i}+1\right)=\sum_{j=1}^{k} \sum_{i=1}^{m_{j}}\left\{k\left(\beta_{i}^{(j)}-i+1\right)-j\right\}+\sum_{i=1}^{q k+r} i \\
& =k\left(\left|\beta^{(1)}\right|+\cdots+\left|\beta^{(k)}\right|\right)+\sum_{i=1}^{q k+r} i-\sum_{j=1}^{k} \sum_{i=1}^{m_{j}}\{k(i-1)+j\} \\
& =k n,
\end{aligned}
$$

since $k(i-1)+j$ takes each value in $\{1, \ldots, q k+r\}$ as $(i, j)$ varies over its range. Thus $\beta^{\vee} \vdash k n$. Moreover, it is clear from the construction that $\beta^{\vee}$ is $k$-balanced.

Lemma 4.6. $\Delta_{k}: \mathscr{B}_{k, n} \rightarrow \Pi_{k, n}: \theta \mapsto \theta^{\wedge}$ is bijective.

Proof. It is now a matter of straightforward calculation with Definitions 4.1 and 4.4 to establish that $\left(\theta^{\wedge}\right)^{\vee}=\theta$ for all $\theta \in \mathscr{B}_{k, n}$, and $\left(\phi^{\vee}\right)^{\wedge}=\phi$ for all $\phi \in \Pi_{k, n}$.

We call $\Delta_{k}(\theta)$ the (ordered) $k$-factorization of $\theta \in \mathscr{B}_{k, n}$. Appendix A gives the 2-factorizations for $\mathfrak{S}_{2}$ and $\mathfrak{S}_{4}$, and the 2- and 3-factorizations for $\mathfrak{S}_{6}$. For example, it is seen from this that $\left[\begin{array}{lll}3 & 2 & 1\end{array}\right]$ is not 2-balanced and that the remaining partitions of 6 are in one-to-one correspondence with $\Pi_{2,3}$. In addition, we give an instance of the connection between an $a$-factorization and an $a b$-factorization. The 2 - and 4-factorizations of $\left[\begin{array}{ll}3^{2} & 2^{2} \\ \left.1^{2}\right]\end{array}\right]$ are $\left(\left[1^{2}\right],\left[21^{2}\right]\right)$ and $\left(\varnothing,\left[1^{2}\right],[1], \varnothing\right)$, respectively. The latter is the interleaving of $(\varnothing,[1])$ and $\left(\left[1^{2}\right], \varnothing\right)$, the 2-factorizations (see Appendix A) of $\left[1^{2}\right]$ and $\left[21^{2}\right]$, respectively, to the first and third positions and the second and fourth positions.

Definition 4.7. Let $\pi_{\alpha}, \pi_{\alpha}^{\prime} \in \mathfrak{S}_{l(\alpha)}$ be defined by

$$
\begin{aligned}
& \pi_{\alpha}=\left(\begin{array}{ccccccc}
1 & 2 & \cdots & m_{1} & m_{1}+1 & \cdots & l(\alpha) \\
\eta_{1,1} & \eta_{1,2} & \cdots & \eta_{1, m_{1}} & \eta_{2,1} & \cdots & \eta_{k, m_{k}}
\end{array}\right) \text {, } \\
& \pi_{\alpha}^{\prime}=\left(\begin{array}{cccccc}
1 & 2 & 3 & \cdots & \cdots & l(\alpha) \\
1 & k+1 & 2 k+1 & \cdots & 2 k+2 & \cdots
\end{array}\right)
\end{aligned}
$$

where $\eta_{s, t}$ and $m_{t}$ are given in Definition 4.1(1). Then $\pi_{\alpha}$ and $\pi_{\alpha}^{\prime}$ are called the associated permutations of $\alpha^{\wedge}$.

With these preliminary results, we may now give the desired factorization of $\chi^{\theta}$ at a semiregular element.

Lemma 4.8. Let $\theta \vdash a b n$. If $\theta$ is not a-balanced, then $\chi_{\left[(a b)^{n}\right]}^{\theta}=0$. Otherwise let $\Delta_{a}(\theta)=\left(\theta^{(1)}, \ldots, \theta^{(a)}\right)$ with associated permutations $\pi_{\theta}, \pi_{\theta}^{\prime}$, and let $n_{s}=$ $\left|\theta^{(s)}\right| / b$ for $s=1, \ldots, a$. Then

$$
\chi_{\left[(a b)^{n}\right]}^{\theta}=\operatorname{sgn}\left(\pi_{\theta} \pi_{\theta}^{\prime}\right) n ! \prod_{s=1}^{a} \frac{1}{n_{s} !} \chi_{\left[b^{n_{s}}\right]}^{\theta^{(s)}},
$$

with the convention that $\chi_{\left[b^{n_{s}}\right]}^{\theta^{(s)}}=0$ if $n_{s} \notin \mathscr{N}$. 
Proof. Since the $p_{\alpha}$ 's form a basis of $\Lambda$,

$$
\Xi_{k}: \Lambda \rightarrow \mathbf{Q}[[t]]: p_{\alpha} \mapsto t^{k r} \delta_{\alpha,\left[k^{r}\right]},
$$

extended linearly to $\Lambda$, is a ring homomorphism. Thus, from the results of Jacobi and Frobenius given at the beginning of this section,

$$
\begin{aligned}
\chi_{\left[(a b)^{n}\right]}^{\theta} & =g^{-1}\left(\left[(a b)^{n}\right]\right)\left[p_{a b}^{n}\right] s_{\theta} \\
& =(a b)^{n} n !\left[p_{a b}^{n}\right]\left\|h_{\theta_{i}-i+j}\right\|_{l(\theta) \times l(\theta)} \\
& =(a b)^{n} n !\left[t^{n a b}\right] \Xi_{a b}\left\|h_{\theta_{i}-i+j}\right\|_{l(\theta) \times l(\theta)} \\
& =(a b)^{n} n !\left[t^{n a b}\right]\left\|\Xi_{a b} h_{\theta_{i}-i+j}\right\|_{l(\theta) \times l(\theta)}
\end{aligned}
$$

But

$$
\begin{aligned}
\Xi_{a b} h_{r} & =\left[v^{r}\right] \Xi_{a b} \prod_{i \geq 1}\left(1-v x_{i}\right)^{-1}=\left[v^{r}\right] \Xi_{a b} \exp \sum_{i \geq 1} p_{i} v^{i} / i \\
& =\frac{t^{r}}{(a b)^{r / a b}(r / a b) !} \tau_{a b}(r)
\end{aligned}
$$

where $\tau_{j}(r)=1$ if $r \equiv 0 \bmod j$ and is 0 otherwise. Since $\theta \vdash a b n$,

$$
\chi_{\left[(a b)^{n}\right]}^{\theta}=(a b)^{n} n !\left\|\frac{\tau_{a b}\left(\theta_{i}-i+j\right)}{(a b)^{\left(\theta_{i}-i+j\right) / a b}\left(\left(\theta_{i}-i+j\right) / a b\right) !}\right\|_{l(\theta) \times l(\theta)}=n !|\mathbf{M}|
$$

where

$$
\mathbf{M}=\left\|\frac{\tau_{a b}\left(\theta_{i}-i+j\right)}{\left(\left(\theta_{i}-i+j\right) / a b\right) !}\right\|_{l(\theta) \times l(\theta)}
$$

Whether or not $\theta$ is $a$-balanced, we can still define $\theta^{\wedge}=\left(\theta^{(1)}, \ldots, \theta^{(a)}\right)$ by setting $k=a$ in Definition 4.1. Thus $m_{s}$ and $\eta_{i, s}$ are determined. The permutations $\rho=\pi_{\theta}^{\prime}$ acting on the rows of this matrix and $\sigma=\pi_{\theta}$ acting on its columns (have been constructed to) rearrange the matrix as a direct sum while preserving the determinant, of course, up to sign. Thus

$$
\chi_{\left[(a b)^{n}\right]}^{\theta}=\operatorname{sgn}(\sigma \rho) n !\left|\mathbf{M}_{1} \oplus \cdots \oplus \mathbf{M}_{a}\right|
$$

where

$$
\left[\mathbf{M}_{1} \oplus \cdots \oplus \mathbf{M}_{a}\right]_{i j}=\frac{\tau_{a b}\left(\theta_{\rho(i)}-\rho(i)+\sigma(j)\right)}{\left(\left(\theta_{\rho(i)}-\rho(i)+\sigma(j)\right) / a b\right) !}
$$

for $1 \leq i, j \leq l(\theta)$, and $\mathbf{M}_{s}$ is $m_{s} \times\lceil(l(\theta)-s+1) / a\rceil$. To see this, it is sufficient to note that $\tau_{a b}\left(\theta_{\rho(i)}-\rho(i)+\sigma(j)\right)=0$ for any $(i, j)$ not corresponding to an element position in one of the blocks $\mathbf{M}_{1}, \ldots, \mathbf{M}_{a}$ of the direct sum, since $\theta_{\rho(i)}-\rho(i)+\sigma(j) \not \equiv 0 \bmod a$ for such values of $(i, j)$. 

where

If $\theta$ is $a$-balanced, then $\mathbf{M}_{s}$ is square so $\chi_{\left[(a b)^{n}\right]}^{\theta}=\operatorname{sgn}(\sigma \rho) n ! \prod_{s=1}^{a}\left|\mathbf{M}_{s}\right|$

$$
\begin{aligned}
{\left[\mathbf{M}_{s}\right]_{i k} } & =\frac{\tau_{a b}\left(\theta_{\eta_{s, i}}-\eta_{s, i}+(k-1) a+s\right)}{\left(\left(\theta_{\eta_{s, i}}-\eta_{s, i}+(k-1) a+s\right) / a b\right) !} \\
& =\frac{\tau_{a b}\left(a\left(\theta_{i}^{(s)}-i+k\right)\right)}{\left(\left(\theta_{i}^{(s)}-i+k\right) / b\right) !}=\frac{\tau_{b}\left(\theta_{i}^{(s)}-i+k\right)}{\left(\left(\theta_{i}^{(s)}-i+k\right) / b\right) !} .
\end{aligned}
$$

Since $\mathbf{M}_{s}$ has the same form as $\mathbf{M}$, we can evaluate $\left|\mathbf{M}_{s}\right|$ by trying to find a $b$-factorization of $\theta^{(s)}$ and then using its associated permutations to rearrange $\mathbf{M}_{s}$. If $n_{s} \notin \mathscr{N}$, then from Proposition $4.3, \theta^{(s)}$ is not $b$-balanced. Then, using the same argument that was used to show that $|\mathbf{M}|=0$ when $\theta$ is not $a$-balanced, we have $\left|\mathbf{M}_{s}\right|=0$. If $n_{s} \in \mathcal{N}$, then $\left|\mathbf{M}_{s}\right|=n_{s} !^{-1} \chi_{\left[b^{n_{s}}\right]}^{\theta^{(s)}}$ from (1) and this completes the proof.

An immediate consequence of Lemma 4.6 and Lemma 4.8 is that

$$
\left|\left\{\theta \vdash p n: \chi_{\left[p^{n}\right]}^{\theta} \neq 0\right\}\right|=\left[q^{n}\right] \prod_{i \geq 1}\left(1-q^{i}\right)^{-p} .
$$

From the Jacobi triple product identity [1] we have $\left|\left\{\theta \vdash 3 n: \chi_{\left[3^{n}\right]}^{\theta} \neq 0\right\}\right|=$ $\left[q^{n}\right]\left\{\sum_{k \geq 0}(-1)^{k}(2 k+1) q^{\left(\begin{array}{c}k+1 \\ 2\end{array}\right)}\right\}^{-1}$.

When $b=1$, then $\theta \vdash n a$ and Lemma 4.8 gives

$$
\chi_{\left[a^{n}\right]}^{\theta}=\operatorname{sgn}\left(\pi_{\theta} \pi_{\theta}^{\prime}\right)\left[\begin{array}{c}
n \\
n_{1}, \ldots, n_{a}
\end{array}\right] \prod_{j=1}^{a} f^{\theta^{(j)}} .
$$

This appears in [15], where it is proved by means of p-cores and p-quotients of tableaux, in conjunction with the Murnaghan-Nakayama rule.

The $a$-factorization of $\tilde{\theta}$ can be deduced from the $a$-factorization of $\theta$.

Proposition 4.9. Let $\left(\theta^{(1)}, \ldots, \theta^{(a)}\right)$ be the a-factorization of $\theta$. Then $\left(\tilde{\theta}^{(a)}\right.$, $\left.\ldots, \tilde{\theta}^{(1)}\right)$ is the a-factorization of $\tilde{\theta}$.

The expression $\prod_{1 \leq i \leq l(\theta)}(x-k i+k)^{\left(k \theta_{i}\right)}$, which is related to $H_{\theta}$, has a particularly useful form when it is reexpressed in terms of the $a$-factorization of $\theta$. A preliminary result is required.

Proposition 4.10. Let $\mathscr{I} \subseteq \mathcal{N}$ and let $a_{i}, b_{i} \in \mathcal{N}$ be such that $a_{i} \leq b_{i}$ for all $i \in \mathscr{I}$. Let $\pi$ be a permutation on the elements of $\mathscr{I}$ such that $a_{\pi(i)} \leq b_{i}$ for all $i \in \mathscr{I}$. Then, for any fixed $c \in \mathcal{N}$

$$
\left|\left\{i \in \mathscr{I}: a_{\pi(i)} \leq c \leq b_{i}\right\}\right|=\left|\left\{i \in \mathscr{I}: a_{i} \leq c \leq b_{i}\right\}\right|
$$

Proof. The left-hand side is

$$
\begin{aligned}
\left|\left\{i \in \mathscr{I}: a_{\pi(i)} \leq c\right\}\right|-\mid\left\{i \in \mathscr{I}: a_{\pi(i)} \leq c \text { and } b_{i}<c\right\} \mid \\
\quad=\left|\left\{i \in \mathscr{J}: a_{\pi(i)} \leq c\right\}\right|-\left|\left\{i \in \mathscr{I}: b_{i}<c\right\}\right| \\
\quad=\left|\left\{i \in \mathscr{I}: a_{i} \leq c\right\}\right|-\left|\left\{i \in \mathscr{I}: b_{i}<c\right\}\right|
\end{aligned}
$$


since $a_{\pi(i)} \leq c$ is forced by $b_{i}<c$, and this then gives the right-hand side.

Lemma 4.11. Let $H_{k}^{\theta}(x)=\prod_{1 \leq i \leq l(\theta)}(x-k i+k)^{\left(k \theta_{i}\right)}$. Then

$$
H_{1}^{\theta}(x)=H_{k}^{\theta^{(1)}}(x) H_{k}^{\theta^{(2)}}(x-1) \cdots H_{k}^{\theta^{(k)}}(x-k+1)
$$

whenever $\theta$ is $k$-balanced and $\left(\theta^{(1)}, \ldots, \theta^{(k)}\right)$ is its $k$-factorization.

Proof. From Definition 4.1(1)

$$
\begin{aligned}
H_{1}^{\theta}(x) & =\prod_{1 \leq i \leq l(\theta)}(x-i+1)^{\left(\theta_{i}\right)}=\prod_{j=1}^{k} \prod_{1 \leq i \leq l\left(\theta^{(j)}\right)}\left(x-\eta_{j, i}+1\right)^{\left(\theta_{\eta_{j, i}}\right)} \\
& =\prod_{j=1}^{k} \prod_{1 \leq i \leq l\left(\theta^{(j)}\right)}\left(x-\eta_{j, i}+1\right)^{\left(k \theta_{i}^{(j)}-k i+k+\eta_{j, i}-j\right)}
\end{aligned}
$$

whence

$$
H_{k}^{\theta^{(1)}}(x) \cdots H_{k}^{\theta^{(k)}}(x-k+1)=\prod_{j=1}^{k} \prod_{1 \leq i \leq l\left(\theta^{(j)}\right)}(x-j+1-k i+k)^{\left(k \theta_{i}^{(j)}\right)} .
$$

Thus $H_{1}^{\theta}(x)=\prod_{r=-\infty}^{+\infty}(x+r)^{u_{r}}$ and $\prod_{j=1}^{k} H_{k}^{\theta^{(j)}}(x-j+1)=\prod_{r=-\infty}^{+\infty}(x+r)^{v_{r}}$ where $u_{r}, v_{r} \geq 0$ for all $r$ and only a finite number of them are nonzero. It remains to prove that $u_{r}=v_{r}$ for all integers $r$. Now

$$
u_{r}=\left|\left\{(i, j): 1-\eta_{j, i} \leq r \leq k \theta_{i}^{(j)}-k i+k-j, 1 \leq j \leq k, 1 \leq i \leq l\left(\theta^{(j)}\right)\right\}\right|
$$

and

$v_{r}=\left|\left\{(i, j): 1-k(i-1)-j \leq r \leq k \theta_{i}^{(j)}-k i+k-j, 1 \leq j \leq k, 1 \leq i \leq l\left(\theta^{(j)}\right)\right\}\right|$.

But $\theta$ is $k$-balanced, so as $(i, j)$ takes permissible values, both $k(i-1)+j$ and $\eta_{j, i}$ take the values $1,2, \ldots, l(\theta)$ exactly once each. Thus $u_{r}=v_{r}$ by Proposition 4.10.

\section{QUADRANGULATIONS OF ARBITRARY GENUS}

We can now give the first of the combinatorial applications of this theory by deriving a bijection between the sets of all rooted quadrangulations of genus $g$ and a set of rooted maps of genus less than or equal to $g$. The result is a consequence of a particular factorization of $R_{\{4\}}$. Other bijections are given in [14].

\section{Theorem 5.1.}

$$
R_{\{4\}}(x, y \mid z)=R_{\mathscr{N}}\left(\frac{1}{2} x, \frac{1}{2}(x+1) \mid 4 z^{2} y\right) R_{\mathscr{N}}\left(\frac{1}{2}(x-1), \frac{1}{2} x \mid 4 z^{2} y\right) .
$$

Proof. From Corollary 3.8(1)

$$
R_{\{4\}}(x, y \mid z)=\sum_{n \geq 0} \frac{z^{2 n}}{(2 n) !} \frac{y^{n}}{4^{n} n !} \sum_{\theta \vdash 4 n} \chi_{\left[4^{n}\right]}^{\theta} \chi_{\left[2^{2 n}\right]}^{\theta} H_{\theta}(x) .
$$


Let $\left(\theta^{(1)}, \theta^{(2)}\right)$ be the 2-factorization of $\theta$. From Lemma 4.8, the second summation may be restricted to 2-balanced $\theta$ for which $\left|\theta^{(1)}\right|$ and $\left|\theta^{(2)}\right|$ are even, for otherwise $\chi_{\left[4^{n}\right]}^{\theta}=0$. With $\left|\theta^{(1)}\right|=2 k$ and $\left|\theta^{(2)}\right|=2 n-2 k$, Lemma 4.8 gives

$$
\begin{aligned}
& \chi_{\left[4^{n}\right]}^{\theta}=\operatorname{sgn}\left(\pi_{\theta} \pi_{\theta}^{\prime}\right)\left(\begin{array}{l}
n \\
k
\end{array}\right) \chi_{\left[2^{k}\right]}^{\theta^{(1)}} \chi_{\left[2^{n-k}\right]}^{\theta^{(2)}}, \\
& \chi_{\left[2^{2 n}\right]}^{\theta}=\operatorname{sgn}\left(\pi_{\theta} \pi_{\theta}^{\prime}\right)\left(\begin{array}{l}
2 n \\
2 k
\end{array}\right) f^{\theta^{(1)}} f^{\theta^{(2)}} .
\end{aligned}
$$

Moreover, from Lemma 4.11, $\prod_{1 \leq i \leq l(\theta)}(x-i+1)^{\left(\theta_{i}\right)}=H_{2}^{\theta^{(1)}}(x) H_{2}^{\theta^{(2)}}(x-1)$. It is known from Lemma 4.6 that $\Delta_{k}: \mathscr{B}_{2, n} \rightarrow \Pi_{2, n}$ is a bijection, so the above summation can now be transformed into

$$
\begin{aligned}
R_{\{4\}}(x, y \mid z)= & \sum_{n \geq 0} \frac{z^{2 n}}{(2 n) !} \frac{y^{n}}{4^{n} n !} \sum_{\substack{k=0 \\
k}}^{n}\left(\begin{array}{l}
n \\
k
\end{array}\right)\left(\begin{array}{l}
2 n \\
2 k
\end{array}\right) \\
& \times \sum_{\substack{\theta^{(1)}-2 k \\
\theta^{(2)}-2 n-2 k}} \chi_{\left[2^{k}\right]}^{\theta^{(1)}} \chi_{\left[2^{n-k}\right]}^{(2)} f^{\theta^{(1)}} f^{\theta^{(2)}} H_{2}^{\theta^{(1)}}(x) H_{2}^{\theta^{(2)}}(x-1) \\
= & A(x, y \mid z) A(x-1, y \mid z)
\end{aligned}
$$

where

$$
A(x, y \mid z)=\sum_{\substack{\theta \\|\theta| \equiv 0 \bmod 2}} \frac{z^{|\theta|} y^{(1 / 2)|\theta|}}{2^{|\theta|}\left(\frac{1}{2}|\theta|\right) !|\theta| !} \chi_{\left[2^{(1 / 2)|\theta|}\right]}^{\theta} f^{\theta} H_{2}^{\theta}(x) .
$$

To identify $A(x, y \mid z)$, note that

$$
\begin{aligned}
H_{2}^{\theta}(x) & =\prod_{1 \leq i \leq l(\theta)}(x-2 i+2)^{\left(2 \theta_{i}\right)} \\
& =\prod_{1 \leq i \leq l(\theta)} 2^{2 \theta_{i}}\left(\frac{1}{2} x-i+1\right)^{\left(\theta_{i}\right)}\left(\frac{1}{2}(x+1)-i+1\right)^{\left(\theta_{i}\right)}
\end{aligned}
$$

so, from Corollary 3.5(1), $A(x, y \mid z)=R_{\mathscr{N}}\left(\frac{1}{2} x, \frac{1}{2}(x+1) \mid 4 z^{2} y\right)$, which gives the result.

The next corollary gives the bijection.

Corollary 5.2. Let $M_{\mathscr{A}}^{(g)}(x, y, z)=\left[u^{2 g}\right] M_{\mathscr{A}}\left(u^{2}, x, y, z\right)$. Then $M_{\{4\}}\left(u^{2}, x, y, z\right)=\frac{1}{2}\left\{M_{\mathscr{N}}\left(4 u^{2}, x+u, x, z^{2} y\right)+M_{\mathscr{N}}\left(4 u^{2}, x-u, x, z^{2} y\right)\right\}$,

$$
M_{\{4\}}^{(g)}(x, y, z)=\left.\sum_{i=0}^{g} \frac{4^{i}}{(2 g-2 i) !} \frac{\partial^{2 g-2 i}}{\partial x^{2 g-2 i}} M_{\mathcal{N}}^{(i)}(x, y, z)\right|_{\substack{y \mapsto x \\ z \mapsto z^{2} y}} .
$$


Proof. (1) From Theorem 3.2(1),

$$
\begin{aligned}
M_{\{4\}}\left(u^{2}, x, y, z\right) & =\Omega_{u} \log R_{\{4\}}(x, y \mid z) \\
& =2 u^{2} z \frac{\partial}{\partial z} \log R_{\{4\}}\left(x u^{-1}, y u^{-1} \mid \frac{1}{2} z u\right)
\end{aligned}
$$

so, from Theorem 5.1,

$$
\begin{aligned}
M_{\{4\}}\left(u^{2}, x, y, z\right)= & 2 u^{2} z \frac{\partial}{\partial z} \log R_{\mathscr{N}}\left(\frac{1}{2} x u^{-1}, \frac{1}{2}\left(x u^{-1}+1\right) \mid z^{2} y u\right) \\
& +2 u^{2} z \frac{\partial}{\partial z} \log R_{\mathscr{N}}\left(\frac{1}{2}\left(x u^{-1}-1\right), \frac{1}{2} x u^{-1} \mid z^{2} y u\right) .
\end{aligned}
$$

Let $\log R_{\mathscr{N}}(x, y \mid z)=\sum_{i, j, k \geq 0} c_{i, j, k} x^{i} y^{j} z^{k} / k !$. Then

$$
\begin{aligned}
M_{\{4\}}\left(u^{2}, x, y, z\right) \\
=\sum_{i, j, k \geq 0} c_{i, j, k} \frac{z^{2 k}}{(k-1) !} \frac{y^{k}}{2^{i+j-2}} u^{k-i-j+2}\left(x^{i}(x+u)^{j}+x^{j}(x-u)^{i}\right) \\
\quad=\frac{1}{2} \sum_{i, j, k \geq 0} c_{i, j, k} \frac{z^{2 k}}{2^{k-1}(k-1) !} y^{k}(2 u)^{k-i-j+2}\left(x^{i}(x+u)^{j}+x^{j}(x-u)^{i}\right) \\
=\frac{1}{2}\left\{M_{\mathscr{N}}\left(4 u^{2}, x, x+u, z^{2} y\right)+M_{\mathscr{N}}\left(4 u^{2}, x-u, x, z^{2} y\right)\right\}
\end{aligned}
$$

from Lemma 2.3. The result now follows from Proposition 3.1.

(2) From (1)

$$
\begin{aligned}
M_{\{4\}}^{(g)}(x, y, z) & =\frac{1}{2}\left[u^{2 g}\right]\left\{M_{\mathscr{N}}\left(4 u^{2}, x+u, x, z^{2} y\right)+M_{\mathcal{N}}\left(4 u^{2}, x-u, x, z^{2} y\right)\right\} \\
& =\left[u^{2 g}\right] M_{\mathscr{N}}\left(4 u^{2}, x+u, x, z^{2} y\right) \\
& =\sum_{i=0}^{g} 4^{i}\left[u^{2 g-2 i}\right] M_{\mathscr{N}}^{(i)}\left(x+u, x, z^{2} y\right)
\end{aligned}
$$

and the result follows.

When $g=0$ we obtain $M_{\{4\}}^{(0)}(x, y, z)=M_{\mathcal{N}}^{(0)}\left(x, x, z^{2} y\right)$, a generating function equation corresponding to the following known explicit bijection [18] between rooted quadrangulations on $2 n$ edges in the sphere and rooted maps on $n$ edges in the sphere. Consider a rooted map $\mu$ and let $\mathscr{V}$ be its vertex set. Now put a vertex in the interior of each face of $\mu$. Let $\mathscr{V}^{\prime}$ denote the set of these new vertices. In traversing the boundary of each face, join each vertex encountered to the vertex in the face. Now consider the tail $v$ of the root edge of $\mu$, and find the first edge $e^{\prime}$ not in $\mu$ encountered in a direction consistent with the orientation of the sphere. Delete the edges of $\mu$ and direct the edge $e^{\prime}$ so that its tail is $v$. Designate this to be the rooting of the resulting map $\mu^{*}$. This map is called the medial of $\mu$, and it is 4-face-regular. The construction is reversible and it gives the desired bijection. Each edge in $\mu^{*}$ joins a vertex in $\mathscr{V}$ to a vertex in $\mathscr{V}^{\prime}$, so $\mu^{*}$ is a bipartite map. In the sphere, all 4-face-regular 


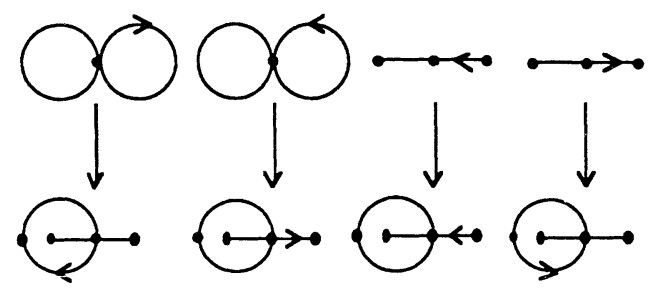

FIGURE 3. Rooted maps and their medials

maps are bipartite. Figure 3 gives four rooted maps, and below them, their medials. The construction is not bijective if the rooting is not made, since dual maps have the same medial.

To see that the construction is reversible, note that the root vertex of $\mu^{*}$ is the root vertex $v$ of $\mu$. The associated graph is bipartite with vertex partition $\left(\mathscr{V}, \mathscr{V}^{\prime}\right)$, with $v \in \mathscr{V}$. In traversing the boundary of each face of $\mu^{*}$ exactly two (possibly identical) vertices of $\mathscr{V}$ are encountered. These are joined to form the edge set of $\mu$.

In surfaces of higher genus, the medial construction extends to a bijection between the set of all rooted maps and the set of all bipartite 4-face-regular rooted maps, a fact which is easily proved both combinatorially and by this (character theoretic) approach. However, both (1) and (2) of the above corollary suggest combinatorial bijections between the set of rooted maps and the set of all 4-face-regular rooted maps. Note that $m !^{-1}\left(\partial^{m} / \partial x^{m}\right) M_{\mathcal{N}}^{(g)}(x, y, z)$ in (2) is the generating function for all rooted maps of genus $g$, with an unordered set of $m$ marked vertices (the hollow vertices in Figure 4), with respect to the number of edges, faces, and unmarked vertices. In (1), the argument $x+u$ suggests that there may be a combinatorial bijection in which handles, marked by $u$, in some way behave as vertices (marked by $x$ ).

The bijection can be illustrated readily in a small example by listing the 15 rooted quadrangulations, with 4 edges, in the torus. These are shown in Figure $4(\mathrm{a})$. Now the number of such maps is, by definition, $\left[z^{4}\right] M_{\{4\}}^{(1)}(1,1, z)$, and from Corollary 5.2(2), with $g=1$, this is equal to

$$
\left.\left[z^{4}\right] \frac{1}{2} \frac{\partial^{2}}{\partial x^{2}} M_{\mathcal{N}}^{(0)}\left(x, 1, z^{2}\right)\right|_{x=1}+\left[z^{4}\right] 4 M_{\mathscr{N}}^{(1)}\left(1,1, z^{2}\right),
$$

which is now interpreted combinatorially.

In (2), the term $\left.\left[z^{4}\right] \frac{1}{2}\left(\partial^{2} / \partial x^{2}\right) M_{\mathcal{N}}^{(0)}\left(x, 1, z^{2}\right)\right|_{x=1}$ is the number of rooted maps with two edges in the sphere, having exactly two marked vertices (shown as hollow), counted with multiplicity 1 . Figure $4(\mathrm{~b})$ gives the 11 such maps (the multiplicity is in parentheses). Thus the contribution to (2), with regard to multiplicity, is 11 . In addition, the term $\left[z^{4}\right] 4 M_{\mathcal{N}}^{(1)}\left(1,1, z^{2}\right)$ is the number of rooted maps on two edges in the torus, each counted with multiplicity 4 . Figure 4(b) shows the single such map, whose contribution to (2), with regard 

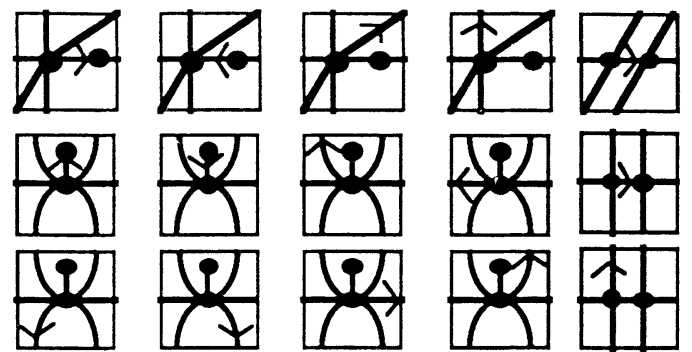

(a) rooted quadrangulations, with 4 edges, in the torus

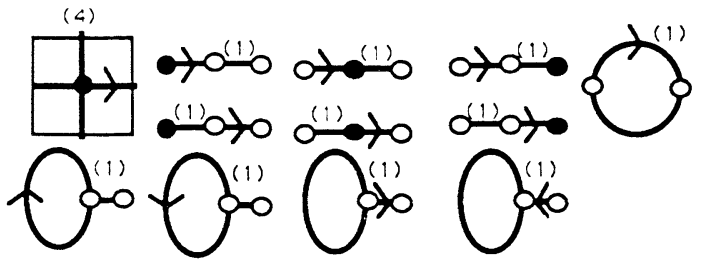

(b) rooted maps, on 2 edges, in the torus and the sphere

FIGURE 4. The bijection for quadrangulations in the torus

to multiplicity, is therefore 4 . The sum of these contributions to (2) is therefore 15 , in agreement with the listing of the rooted quadrangulations with 4 edges in the torus.

\section{Concluding COMMENTS}

$M_{\mathscr{N}}(u, x, y, z)$ has been expressed in terms of a summation, over partitions, of an expression whose dependence on character evaluations is through the degree, $f^{\theta}$, only, for which there is an explicit expression. For $\left[z^{n}\right] M_{\mathcal{N}}(u, x, y, z)$, the summation is over all partitions of $n$.

Decompositions of rooted maps in surfaces of arbitrary genus are uncommon. It would therefore be of interest to have a combinatorial explanation of Corollary 5.2.

Bender and Canfield [3] have proved the following result. Let $\rho=\sqrt{1-12 z}$. Then the generating function for maps of genus $g$ with respect to the number of edges is

$$
\left[u^{g}\right] M_{\mathscr{N}}(u, 1,1, z)=\frac{z^{2 g} P_{g}(\rho)}{\rho^{a}(\rho+1)^{2}(\rho+2)^{b}(\rho+5)^{c}}
$$

where $a, b, c$ are nonnegative integers and $P_{g}(\rho)$ is a polynomial in $\rho$. Again, it seems to be a difficult task to prove this algebraically from the character theoretic forms of the generating functions which we have given here.

Certain algebraic tasks appear to be difficult. For example, it is unclear algebraically that $\Omega_{u} \log R_{\mathscr{A}}$ is a formal power series, although combinatorially this is clearly so. Such proofs may reveal points of value for extracting combinatorial information elsewhere in this context. 
A. Factorizations for $\mathfrak{S}_{2}, \mathfrak{S}_{4}$, and $\mathfrak{S}_{6}$

$\begin{array}{ccc}\theta & 2 \text {-factorization } & \chi_{[2]}^{\theta} \\ {\left[1^{2}\right]} & (\varnothing,[1]) & -1 \\ {[2]} & ([1], \varnothing) & 1\end{array}$

$\theta \quad$ 2-factorization $\quad \chi_{\left[2^{2}\right]}^{\theta}$

$\left[1^{4}\right] \quad\left(\varnothing,\left[1^{2}\right]\right) \quad 1$

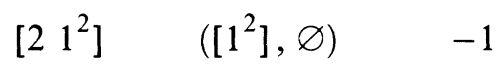

$\left[2^{2}\right] \quad([1],[1]) \quad 2$

[3 1] $\quad(\varnothing,[2]) \quad-1$

[4] $([2], \varnothing) \quad 1$

\begin{tabular}{|c|c|c|c|c|}
\hline $\begin{array}{c}\theta \\
{\left[1^{6}\right]}\end{array}$ & $\begin{array}{l}\text { 2-factorization } \\
\left(\varnothing,\left[1^{3}\right]\right)\end{array}$ & $\begin{array}{c}\chi_{\left[2^{3}\right]}^{\theta} \\
-1\end{array}$ & $\begin{array}{l}\text { 3-factorization } \\
\left(\varnothing, \varnothing,\left[1^{2}\right]\right)\end{array}$ & $\chi_{\left[3^{2}\right]}^{\theta}$ \\
\hline$\left[\begin{array}{ll}2 & 1^{4}\end{array}\right]$ & $\left(\left[1^{3}\right], \varnothing\right)$ & 1 & $\left(\varnothing,\left[1^{2}\right], \varnothing\right)$ & -1 \\
\hline$\left[\begin{array}{ll}2^{2} & 1^{2}\end{array}\right]$ & $\left([1],\left[1^{2}\right]\right)$ & -3 & - & 0 \\
\hline$\left[2^{3}\right]$ & $\left(\left[1^{2}\right],[1]\right)$ & 3 & $(\varnothing,[1],[1])$ & 2 \\
\hline$\left[\begin{array}{ll}3 & 1^{3}\end{array}\right]$ & $\left(\varnothing,\left[\begin{array}{ll}2 & 1\end{array}\right]\right)$ & 2 & $\left(\left[1^{2}\right], \varnothing, \varnothing\right)$ & 1 \\
\hline$\left[\begin{array}{lll}3 & 2 & 1\end{array}\right]$ & - & 0 & $([1], \varnothing,[1])$ & -2 \\
\hline$\left[\begin{array}{ll}4 & 1^{2}\end{array}\right]$ & $([21], \varnothing)$ & -2 & $(\varnothing, \varnothing,[2])$ & 1 \\
\hline$\left[3^{2}\right]$ & $([1],[2])$ & -3 & {$[[1],[1], \varnothing)$} & 2 \\
\hline$\left[\begin{array}{ll}4 & 2\end{array}\right]$ & $([2],[1])$ & 3 & - & 0 \\
\hline$\left[\begin{array}{ll}5 & 1\end{array}\right]$ & $(\varnothing,[3])$ & -1 & $(\varnothing,[2], \varnothing)$ & -1 \\
\hline [6] & $([3], \varnothing)$ & 1 & $([2], \varnothing, \varnothing)$ & \\
\hline
\end{tabular}

\section{REFERENCES}

1. G. E. Andrews, The theory of partitions, Encyclopedia Math. Appl., Vol. 2, Addison-Wesley, Reading, Mass., 1976.

2. D. Arquès, Relations fonctionelles et dénombrement des cartes pointées sur le tore, J. Combinatorial Theory Ser. B 84 (1987), 253-274. 
3. E. A. Bender and E. R. Canfield, The number of rooted maps on an orientable surface (preprint).

4. E. A. Bender and N. C. Wormald, Almost all convex polyhedra are asymmetric, Canad. J. Math. 37 (1985), 854-871.

5. D. Bessis, C. Itzykson, and J. B. Zuber, Quantum field theory techniques in graphical enumeration, Adv. in Appl. Math. 1 (1980), 109-157.

6. J. R. Edmonds, A combinatorial representation for polyhedral surfaces, Notices Amer. Math. Soc. 7 (1960), 646 (Abstract).

7. I. P. Goulden and D. M. Jackson, Combinatorial enumeration, Wiley, New York, 1983.

8. J. L. Gross and S. R. Alpert, The topological theory of current graphs, J. Combinatorial Theory Ser. B 17 (1974), 218-233.

9. J. L. Gross and T. W. Tucker, Topological graph theory, Wiley Interscience, New York, 1987.

10. L. Heffter, Ueber das Problem der Nachbargebiete, Math. Ann. 38 (1891), 477-508.

11. P. Hoffman and B. Richter, Embedding graphs in surfaces, J. Combinatorial Theory Ser. B 36 (1984), 65-84.

12. D. M. Jackson, Counting cycles in permutations by group characters, with an application to a topological problem, Trans. Amer. Math. Soc. 299 (1987), 785-801.

13. _ Some combinatorial problems associated with products of conjugacy classes of the symmetric group, J. Combinatorial Theory Ser. A 49 (1988), 363-369.

14. D. M. Jackson and T. I. Visentin, Character theory and rooted maps on an orientable surface of given genus: face-colored maps, Trans. Amer. Math. Soc. 322 (1990), 365-376.

15. G. James and A. Kerber, The representation theory of the symmetric group, Encyclopedia Math. Appl., Addison-Wesley, Reading, Mass., 1981.

16. I. G. Macdonald, Symmetric functions and Hall polynomials, Clarendon Press, Oxford, 1979.

17. J.-P. Serre, Linear representations of finite groups, Springer-Verlag, New York, 1977.

18. W. T. Tutte, The dissection of equilateral triangles into equilateral triangles, Proc. Cambridge Philos. Soc. 44 (1948), 463-482.

19. __ A census of planar maps, Canad. J. Math. 15 (1963), 249-271.

Department of Combinatorics and Optimization, University of Waterloo, Waterloo, Ontario, Canada N2L 3G1 